\title{
Permeability variation due to sand particles in an infiltration flow using Smoothed Particle Hydrodynamics method
}

\author{
Naoki TANIMOTO $^{1}$, Hitoshi MIKADA ${ }^{1}$ and Junichi TAKEKAWA ${ }^{1}$ \\ ${ }^{1}$ Dept. of Civil and Earth Res. Eng., Kyoto University
}

\begin{abstract}
Recent years, sanding phenomenon makes some problems in resource engineering field. One of the characteristic problems is that small sand particles flow in accumulating layer and close pores of the layer. When the pore closed, sand particles prevent fluids from flowing the pore. Because of that, fluid velocity and permeability decrease. This is big problem for efficient produce of resource, so it is wanted to understand the mechanism for solve the problem. In this paper, we researched the mechanism using simulation by Smoothed Particle Hydrodynamics( $\mathrm{SPH})$ method. As a result, we caught a phenomenon that sand particles close a big pore and fluid velocity decreased dynamically. We simulated in four models which has a difference of shape distribution of sand particles. We found that there is visible difference between permeability changing of these models and shape of sand particles is effective for permeability changing.
\end{abstract}

\section{INTRODUCTION}

In recent years the production management of oil, gas and unconventional reservoirs has been important of real fields. One of the most common problems with the production management is the change in permeability due to small sand particles in porous rocks or production pipe, known as sanding. The increase of the effective stress in the subsurface generates small particles from reservoir rocks (Pinkert et al, 2014 ${ }^{1)}$ ). The permeability of reservoir rock is affected by these particles when those flow through pore spaces of rock to perforation holes. Since the permeability is dynamically decreased because small particles close flow passes, the effect of sanding on the production of hydrocarbon should be evaluated correctly (e.g. Otsuki et al, $2010^{2)}$ ). There are many causes of sanding; viscosity of fluid, pressure gradient, the shape of small sands, and so on.

In the present study, we evaluate the influence of the shape of small particles on permeability change. But, in present study, the shape of small particles is integrated because it is difficult to set boundary conditions of arbitrary shapes of particles. In order to evaluate effect of shape of small particles, we conduct numerical experiments using the Smoothed Particle Hydrodynamics (SPH) method for handling arbitrary shapes of particles (Muller et al,2003 ${ }^{3)}$ ). We construct numerical porous media by SPH particles, and simulate solid-fluid two-phase flow with water and small particles. The shapes of particles vary in circle, square and their mixture. The permeability change due to the behavior of floating particles with different shapes is investigated. Furthermore, we visualize the two-phase flow in order to study the mechanism of permeability changes.

\section{METHOD}

In this study, we use SPH method which is one of particle methods. In this method, solid and fluid are thought of as assemblies of particles. We calculate the behavior of each particle, and capture the macro behavior of multi-phase flow.

Physical quantity of each particle is calcurate by a weighting and gathering quantity of neighbors as shown below.

$$
\mathrm{A}\left(\mathbf{r}_{\mathrm{i}}\right)=\mathrm{m}_{\mathrm{j}}\left(\mathrm{A}\left(\mathbf{r}_{\mathrm{j}}\right) / \rho_{\mathrm{j}}\right) \mathrm{W}(\mathbf{r}, \mathrm{h})
$$

Where $A$ is an arbitrary physical quantity, $r_{i}$ is the position vector of particle $i, m_{j}$ and $\rho_{j}$ are the mass and density of particle $\mathrm{j}$, respectively. $\mathrm{W}$ is a weighting function. $h$ is the radius of an effect domain. We solve the Navier-Stokes equation to simulate incompressible viscous flow.

$$
\mathrm{Du} / \mathrm{Dt}=-(1 / \rho) \nabla \mathrm{P}+v \Delta \mathrm{u}+\mathrm{f} / \rho
$$

The left hand means acceleration, first part of right hand means acceleration pressure gradient, 
second means acceleration from viscosity, third means acceleration from external force. Gradient and Laplacian terms in equation 2 are computed by the summation with the weighting function as follows.

$$
\nabla \mathrm{A}\left(\mathbf{r}_{\mathrm{i}}\right)=\mathrm{m}_{\mathbf{j}}\left(\mathrm{A}\left(\mathbf{r}_{\mathbf{j}}\right) / \rho_{\mathrm{j}}\right) \nabla \mathrm{W}(\mathbf{r}, \mathrm{h})
$$

We assume solid particles as rigid bodies: they don't vary their shape from initial on all time steps in this simulation. Next, we explain rigid bodies in SPH. First, we move SPH particles which construct rigid body the same as SPH particles which constructs fluid. Second, we compute gravity center and rotation angle of a rigid body from the position of SPH particles. Third, we change the position and velocity of each SPH particles so as to maintain the shape of rigid body using gravity center position and rotation angle. We do these three operations in each time step. The fixed position and velocity could affect fluid particles in next time step, so we can compute interaction of solid and fluid.

We also explain interaction between solid particles based on the discrete element method (DEM). If two solid particles are overlapped, the repulsive force acts on these two particles. The magnitude of the repulsive force is computed by multiplying a spring constant by overlapping distance as follows.

$$
\begin{gathered}
\mathbf{F}_{\mathbf{n}}=\mathbf{k}_{\mathbf{n} 1} * \mathbf{d}_{\mathbf{n}}+\mathbf{k}_{\mathbf{n} 2} * \mathbf{U}_{\mathbf{n}} \\
\mathbf{F}_{s}=\mathbf{k}_{s} * \mathbf{U}_{\mathbf{s}}
\end{gathered}
$$

Where $k_{n 1}$ is spring constant for normal direction, $k_{n 2}$ and $k_{s}$ and dashpot contants for normal and shear directions, respectively, $d_{n}$ is overlapping distance in normal directions, $U_{n}$ and $U_{s}$ are velocity variations in normal directions, and shear directions. We show a flow chart of our computation process in Figure 1.

\section{RESULTS}

We set a model of a porous media as shown in Figure 2. The $\quad x$-direction length is $6.5 \mathrm{~mm}$ and the $\quad$-direction length is $1 \mathrm{~mm}$. Black circles placed in the left side of the model are floating rigid bodies, which represent small sand

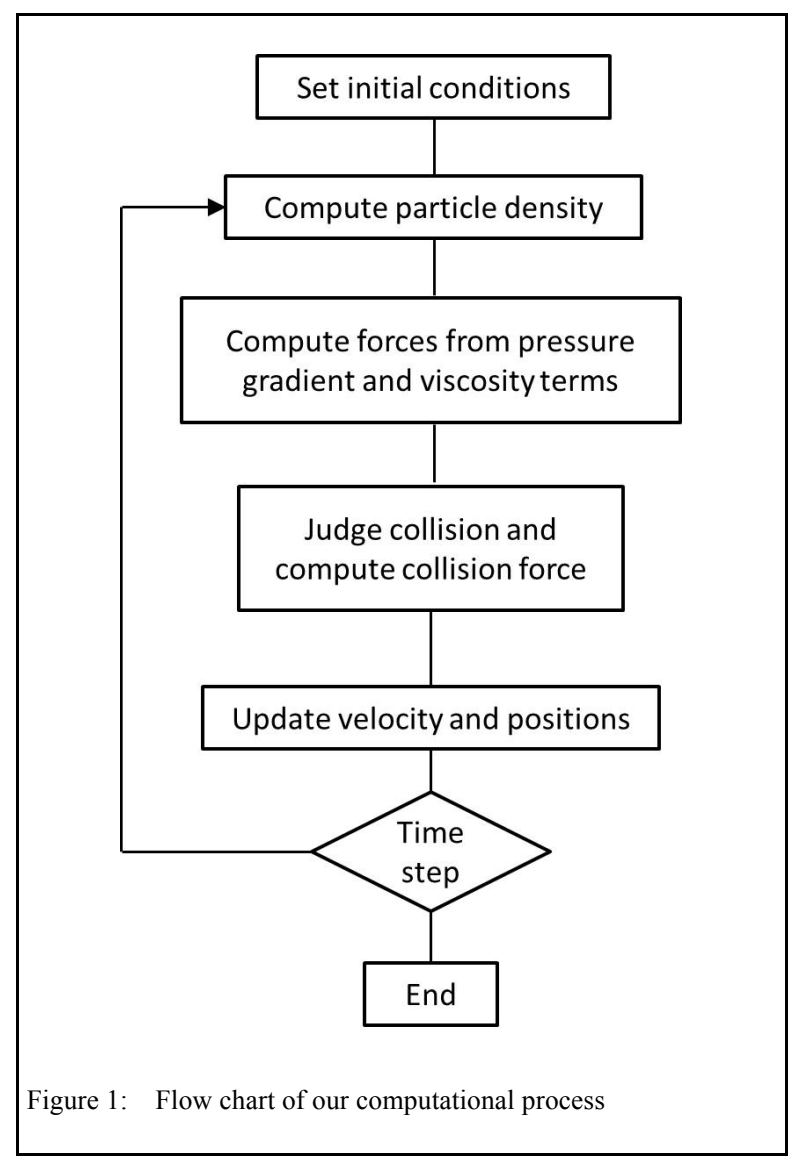

particles. We change the shape of particles to investigate the influence of this to the permeability. The initial shapes are circle and square. White circles placed in the center of the model are immovable rigid bodies which represent the frame of rock. Void spaces between white circles are pores and pore throats. External force, which drives fluid, is applied to every particle except for immovable white circles. Total number of small particles is 20 . We set four different realizations of the arrangement of small particles; 1) 20 circular shaped particles, 2) 20 rectangular shaped particles, 3) 10 circular and 10 rectangular shaped particles, 4) same composition ratio as 3 ) but the location of particles is different. At the one second from the time simulation started, we

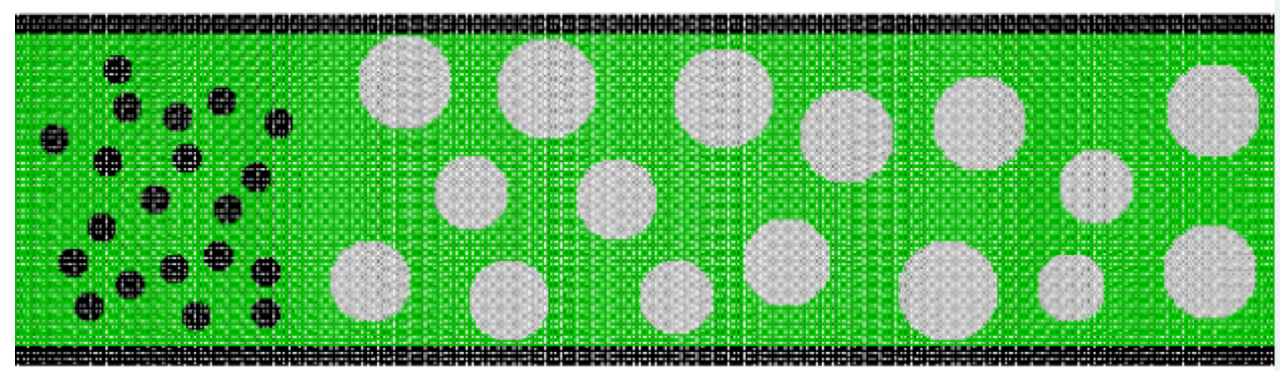

Figure 2: Porous media model. Black and white circles represent movable and non-movable solid. Green particles represent fluid particles. 
move only fluid particles in order to reproduce the steady flow. After that, small particles start to move and pass through the porous media model. We record the change of average velocity along the vertical direction and time variation of permeability of the porous media. Total calculating time is 2.15 second. Figure 3 shows that the velocity distribution averaged along the vertical direction. The shape of each small particle is circle; i.e. case 1). Horizontal axis represents the distance from the left edge. Vertical axis represents the average velocity normalized by the velocity in the porous media without small particles. We show four graphs with different time steps. Velocity graph at $1 \mathrm{~s}$ (red line in Figure 3) shows almost homogeneous distribution of averaging velocity because small particles nave not attached the porous media. At $1.2 \mathrm{~s}$ (blue line in Figure 3), local decrement of average velocity can be observed at the horizontal distance of $0.002 \mathrm{~m}$. Especially at $1.55 \mathrm{~s}$ (pink line in Figure 3) visible reduction can be observed around $0.003 \mathrm{~m}$. This reduction is caused by the blocking of a flow channel due to passage of small particles.

We show snapshots of the two-phase flow in Figure 4. Hot color and cold color represent high and low velocity regions. At 1.2s ((a) in Figure 4),

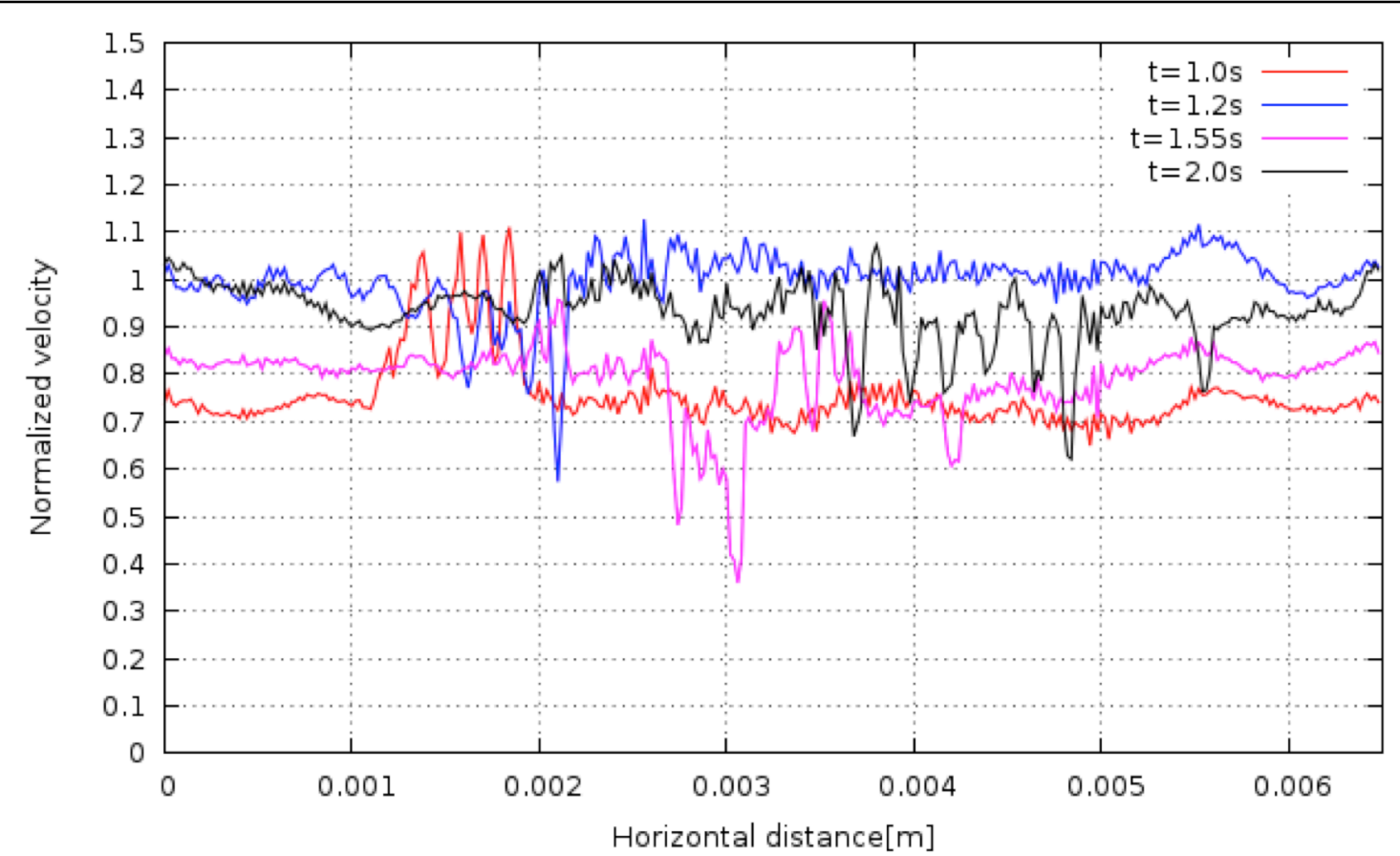

Figure 3: Average velocity in pattern 1 after $1 \mathrm{~s}$ (red), $1.2 \mathrm{~s}$ (blue), $1.55 \mathrm{~s}$ (pink) and $2.0 \mathrm{~s}$ (black)

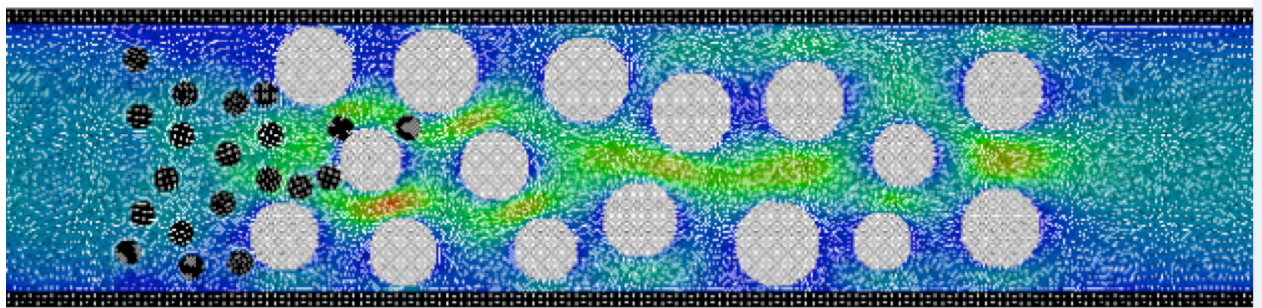

(a) $1.2 \mathrm{~s}$

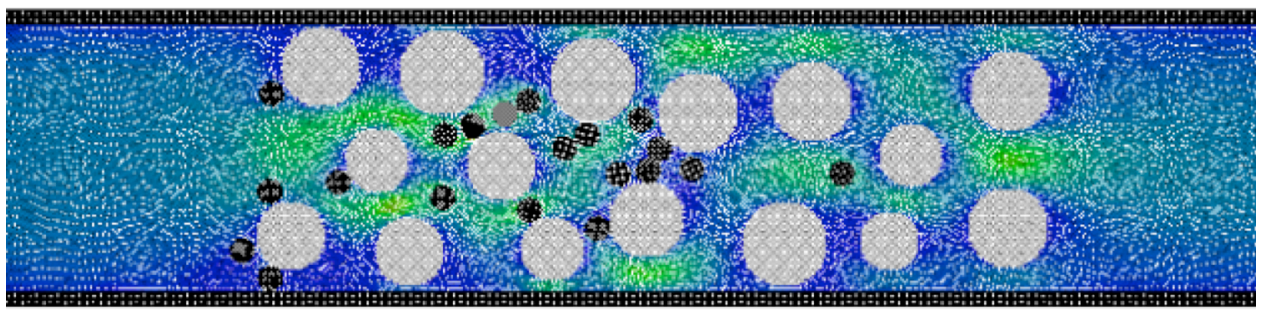

(b) $1.55 \mathrm{~s}$

Figure 4: Snapshots of the motion of small particles after $1.2 \mathrm{~s}$ and $1.55 \mathrm{~s}$. Hot and cold colors represent high and low velocity zone respectively. 
small particles attach the porous media and reduce the width of flow channels. At 1.55s ((b) in Figure 4), small particles pass through the pore throats and fluid velocity are reduced in most of region. Especially at the center of the model, a few small particles completely close the center of the flow. This phenomenon induces the dramatic reduction of the average velocity. This behavior of small particles and interaction with fixed circles could be affected by the shapes of particles in terms of the change in permeability. Permeability of porous media $\mathrm{K}$ is computed by the following equation.

$$
\mathrm{K}=-\mu \mathrm{Q} /\left(\mathrm{A}^{*} \nabla \mathrm{P}\right)
$$

Where $Q$ is the total flowing quantity, $\mu$ is the dynamic viscosity, $\mathrm{P}$ is the pressure. We show the time variations of the permeability in Figure 5 . Vertical axis represents the permeability normalized by the permeability of the porous media without small particles. In the case of 20 circle particles (red line in Figure 5) the permeability decreases around $1.55 \mathrm{~s}$. This stems from the blocking of the flow shown in Figure 4(b). Although the other arrangements do not show any blocking of flow, the difference of the permeability change would be observed. This shows that the shape of small floating particles has significant effect on the permeability change.

\section{CONCLUSIONS}

In the present study, we simulate a flow of solid-fluid two-phase flow in idealized pore space using SPH method in order to investigate the effect of the shape of small floating particles on the evaluation of sanding. We model a porous media by a set of immovable SPH particles in a pipe, and let plural small rigid particles into a stream flowing into the media. Small particles flow, pass through pore-throats and interact with each other. The complete block can be observed in case that all particles are circle, i.e. case pattern 1). In the other cases, complete blocking cannot be observed. The permeability change in time shows that a significant reduction can be observed. Our result shows that the shape of the floating particles has effect on the relative permeability in reservoir rocks. Among any parameter possibly included to simulate sanding, the shape of grains would be also important and should be taken into account for accurate reproduction of the phenomenon.

\section{REFERENCES}

1) Pinkert, S., and Grozic, J. L. H., 2014, Prediction of the mechanical response of hydrate-bearing sands, Journal of Geophysical Research: Solid Earth, 119, 4695-4707.

2) Otsuki, S., and Matsuoka, T., 2010, A study on the Effect of Particle Transport on Permeability in Porous Media by Using Hybrid LBM-DEM Simulation, Journal of MMIJ, 126 (8), 503-511.

3) Muller, M., Charypar, D., and Gross, M., 2003, Particle-Based Simulation for Interactive Applications, Proceeding of the 2003 ACM Eurographics/SIGGRAPH Symposium on Computer Animation, 154-159.

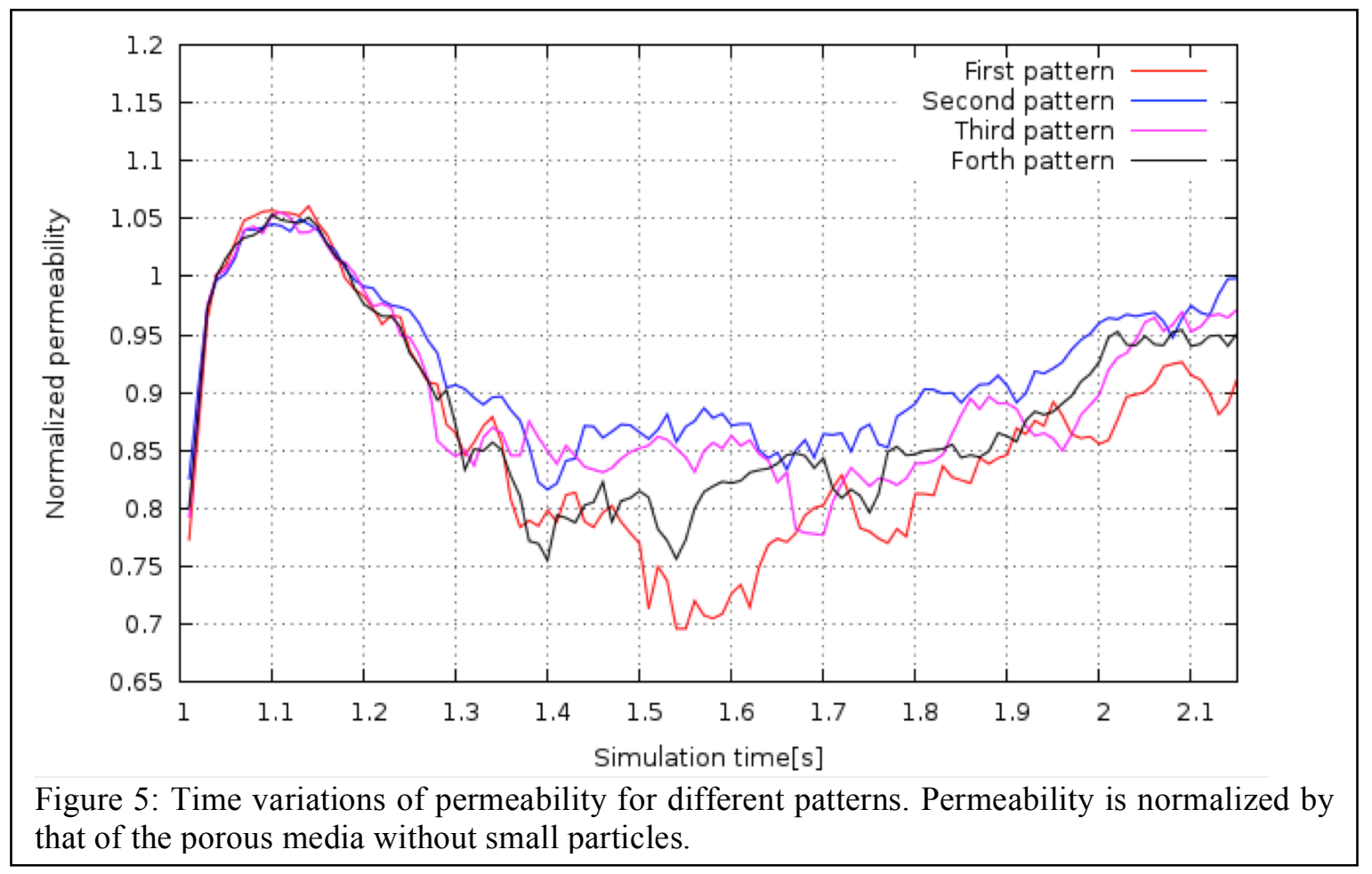

